\title{
Oligopeptide Transporters of the SLC15 Family Are Dispensable for Peptidoglycan Sensing and Transport in Drosophila
}

\author{
Florence Capo Delphine Chaduli Annelise Viallat-Lieutaud Bernard Charroux \\ Julien Royet
}

Aix Marseille Université, CNRS, Institut de Biologie du Développement de Marseille (IBDM), Marseille, France

\section{Keywords}

Drosophila $\cdot$ SLC15 transporters · NF-KB · Peptidoglycan ·

Peptidoglycan recognition proteins

\begin{abstract}
Peptidoglycan (PGN) detection by PGN recognition proteins (PGRP) is the main trigger of the antibacterial immune response in Drosophila. Depending on the type of immune cell, PGN can be sensed either at the cell membrane by PGRP-LC or inside the cell by PGRP-LE, which plays a role similar to that of Nod2 in mammals. Previous work, mainly in cell cultures, has shown that oligopeptide transporters of the SLC15 family are essential for the delivery of PGN for Nod2 detection inside of the cells, and that this function might be conserved in flies. By generating and analyzing the immune phenotypes of loss-of-function mutations in 3 SLC15 Drosophila family members, we tested their role in mediating PGRP-LE-dependent PGN activation. Our results show that Yin, CG2930, and CG9444 are required neither for PGRP-LE activation by PGN nor for PGN transport from the gut lumen to the insect blood. These data show that, while intracellular PGN detection is an essential step of the antibacterial response in both insects and mammals, the types of PGN transporters and sensors are different in these animals.
\end{abstract}

(c) 2017 S. Karger AG, Basel
(C) 2017 S. Karger AG, Basel

\section{KARGER}

E-Mail karger@karger.com

www.karger.com/jin

\section{Introduction}

Antibacterial immune response activation relies on the detection of conserved microbial-associated molecular patterns, such as lipopolysaccharide or peptidoglycan (PGN), by pattern recognition molecules. In mammals, PGN is recognized by PGN recognition proteins (PGRP) and by the Nod-like-receptor family members Nod1 and Nod2 [1]. The cytoplasmic localization of some of these receptors, such as Nod1 and Nod2, raises the question of how PGN produced by extracellular bacteria can gain access to them [2]. Previous work, mainly in cell cultures, has shown that PGN can enter the cytosol following injection through bacterial secretion systems [3] or following delivery by outer membrane vesicles [4]. Nod ligands are also taken up through endocytosis or phagocytosis depending on the cell type $[5,6]$. In addition, oligopeptide transporters, including $\mathrm{pH}$-sensing regulatory factor of peptide transporter 1 (PEPT1, also known as SLC15A1) and solute carrier family 15 member 4 (SLC15A4), are coopted to bring PGN fragments into the cytosol $[5,7]$. In addition, recent work has shown that SLC15A3 and SLC15A4 are preferentially expressed by dendritic cells where they mediate the egress of the Nod2 ligand muramyl-dipeptide from internalized bacteria located inside endolysosomes [8].

Dr. Bernard Charroux or Prof. Julien Royet

Aix Marseille Université, CNRS

Institut de Biologie du Développement de Marseille (IBDM)

FR-13288 Marseille (France)

E-Mail bernard.charroux@univ-amu.fr or julien.royet@univ-amu.fr 
Nod-like receptor orthologs are absent from the Drosophila genome. In this insect, NF- $\kappa \mathrm{B}$ pathway activation by bacteria depends on the previous recognition of PGN by PGRP family members [9-12]. Consequently, PGN detection by PGRP is very versatile in flies, relying on different PGRP proteins that are able to recognize the different structure of PGN at different locations in immune competent cells. PGN derived from Gram-positive bacteria and from bacilli (called Lys-type PGN) is sensed in the circulating insect hemolymph by bloodborne PGRP-SA protein [13]. This interaction is sufficient to activate the Toll signaling pathway. The Drosophila immune surveillance machinery can also detect the presence of di-aminopimelic acid type PGN (DAPPGN) that is present in the cell wall of most Gram-negative bacteria and is also a ligand for mammalian Nod1 [14]. DAP-PGN produced by dividing extracellular bacteria can be detected on the cell surface by the single-pass transmembrane receptor PGRP-LC, leading to IMD/ RIP protein recruitment and NF- $\mathrm{KB}$ signaling activation [15-21]. In addition to PGRP-LC, another PGRP family member, i.e., PGRP-LE, is involved in DAP type PGN recognition. Full-length PGRP-LE does not possess any identifiable transmembrane domain and it is localized in the cytoplasm, where it recognizes DAP-type PGN entering the cytosol, or PGN introduced by cytoinvasive bacteria such as Listeria monocytogenes [16, 17, 22-24]. This dual mode of detection, extracellular via PGRP-LC and intracellular via PGRP-LE, has important physiological implications for the fly response to bacteria. Work from our lab and others has shown that while IMD pathway activation in the fat body (FB) is only dependent on PGRP-LC, PGN detection in gut enterocytes is mainly mediated by the cytoplasmic PGRP-LE sensor $[23,24]$. This enterocyte cytosolic PGN recognition is required to establish both NF- $\kappa B$-dependent responses to infectious bacteria and immune tolerance to microbiota. As for the Nods, the cytosolic localization of PGRP-LE raises the question of how the PGRP-LE ligand derived from the extracellular microbes can access intracellular compartments. This suggests that transport mechanisms that translocate PGN across the plasma membrane and deliver it to the cytosol must exist. Previous in vitro studies have suggested that Drosophila SLC15 family members might also play a role in facilitating PGN entry into the cell. Indeed, ectopic expression of one Drosophila SLC15 family member, i.e., Yin, has been shown to increase the sensitivity of NOD2-dependent NF- $\kappa \mathrm{B}$ activation in mammalian cells [25]. We decided to test the involvement of closely related SLC15 family members in regula- tion of the PGN-dependent Drosophila immune response. For this purpose, we generated loss-of-function alleles for each of the oligopeptide transporters and tested their contribution to the NF- $\kappa \mathrm{B}$ activation triggered by bacterial infection. Our results suggest that none of these proteins is strictly required to facilitate PGN access to cytosolic PGRP-LE. We also demonstrate that these proteins are not involved in the transport of PGN from the gut lumen to the circulating hemolymph (where PGN can have detrimental systemic consequences [26]). In conclusion, these results suggest that vertebrates and invertebrates not only rely on different cytosolic PGN sensors but they also use different mechanisms to transport PGN toward these detectors.

\section{Materials and Methods}

Bacterial Strains

The following microorganisms were used: Erwinia carotovora carotovora 15 pOM1-GFP (Ecc) and Lactobacillus plantarum ${ }^{\text {WJL }}$ (L.p.). Ecc was cultured in Luria-Bertani medium at $30^{\circ} \mathrm{C}$ and $L$. plantarum in MRS medium (Sigma-Aldrich, Agar catalog \#69964 and Broth catalog \#69966) at $37^{\circ} \mathrm{C}$.

Drosophila melanogaster Strains and Maintenance

The following strains were used: $\mathrm{Yin}^{-/-}, \mathrm{CG} 2930^{-/-}, \mathrm{CG} 9444^{-/-}$ (this work), Df(1)ED6716 (BL \#24145), PGRP-LE $E^{112}$ [17], cg-Gal4 (BL\#7011), and UASdFadd ${ }^{I R}[27,28]$. Flies were grown at $25^{\circ} \mathrm{C}$ on a yeast/cornmeal medium. For $1 \mathrm{~L}$ of food, $8.2 \mathrm{~g}$ of agar (VWR, catalog \#20768.361), $80 \mathrm{~g}$ of cornmeal flour (Farigel maize H1; Westhove), and $80 \mathrm{~g}$ of yeast extract (VWR, catalog \#24979.413) were cooked for $10 \mathrm{~min}$ in boiling water; $5.2 \mathrm{~g}$ of methylparaben sodium salt (Merck, catalog \#106756) and $4 \mathrm{~mL}$ of $99 \%$ propionic acid (Carlo Erba, catalog \#409553) were added when the food had cooled to $60^{\circ} \mathrm{C}$. For antibiotic treatment, the standard medium was supplemented with ampicillin, kanamycin, tetracyclin, and erythromycin at a final concentration of $50 \mathrm{mg} / \mathrm{mL}$. For all tests, 6- to 8-day-old females were used.

\section{Mutant Generation}

$\mathrm{Yin}^{-/-}, \mathrm{CG} 2930^{-/-}$, and CG9444-/- KO lines were generated by homologous recombination and each gene was replaced by a miniwhite gene. The primers used to generate homology arms on each side were, respectively: $5^{\prime}$-GGAAATCGACTCGAATATAGGG-3' and $5^{\prime}$-CTGAACTGTGTCCCAAACATTGC- $3^{\prime}$ for the Yin $5^{\prime}$ arm and $5^{\prime}$-CCGTCCATTTGCAAGCTGCGC- $3^{\prime}$ and $5^{\prime}$-CGATAAAGTTATCACATCGG-3' for the Yin $3^{\prime}$ arm, $5^{\prime}$-ATGTGGCTGTGCTAGAGAATTCG- ${ }^{\prime}$ and $5^{\prime}$-GGTACCTGCTCGACGTCGAAGGATGGC- $3^{\prime}$ for the CG2930 $5^{\prime}$ arm and $5^{\prime}$-GAATCTGTCCGCTTATTATATCC- $3^{\prime}$ and $5^{\prime}$-CGACAACAGACTTGATATCATTCG-3' for the CG2930 3' arm, and $5^{\prime}$-TTTACGCTAAATCATTACGC-3' and $5^{\prime}$-ACAGAGCTGCGGCTTTTGTG-3' for the CG9444 $5^{\prime}$ arm and $5^{\prime}$-GTAAGTTTCCAGATTTCCATC-3' and $5^{\prime}$-CAAGGACACGACGGATGTCTC- $3^{\prime}$ for the CG9444 $3^{\prime}$ arm. Sequences were cloned in the $p W 25$ vector [29] for Yin and CG2930 and in the $p T V^{\text {Cherry }}$ vector for CG9444 [30].
484

J Innate Immun 2017;9:483-492

DOI: $10.1159 / 000475771$
Capo/Chaduli/Viallat-Lieutaud/

Charroux/Royet 


\section{Infection of Adults by Ecc}

Overnight bacterial cultures were centrifuged at 4,000 $g$ for 20 min at room temperature and resuspended in fresh Luria-Bertani medium. Cells were serially diluted in PBS and their concentration was determined by optical density (OD) measurement at $600 \mathrm{~nm}$. For oral infection, flies were first incubated for $2 \mathrm{~h}$ at $25^{\circ} \mathrm{C}$ in empty vials and then placed in a fly vial with food. The food solution was obtained by mixing a pellet of an overnight culture of Ecc bacteria $(O D=200)$ with a solution of $5 \%$ sucrose $(50 / 50)$ and added to a filter disk that completely covered the agar surface of the fly vial. Septic injuries were performed by pricking adult females with a thin needle contaminated with Ecc.

Monoassociation of Germ-Free Flies with L. plantarum

Five-day-old germ-free wild-type males (group of 20 flies) were placed in a fly vial with food. The food solution was obtained by mixing a culture of bacteria L. plantarum $(\mathrm{OD}=2)$ with a solution of $5 \%$ sucrose (50/50) and it was added to a filter disk that completely covered the agar surface of the fly vial. Males were incubated at $25^{\circ} \mathrm{C}$ for $24 \mathrm{~h}$ and then placed at $25^{\circ} \mathrm{C}$ in a standard yeast/ cornmeal vial in order to contaminate the vial with L. plantarum containing feces. After 3 days, the males were removed and replaced by 5 -day-old germ-free females (a group of 15 flies). These females were incubated for 3 days at $25^{\circ} \mathrm{C}$ to ensure efficient contamination by L. plantarum before dissection.

\section{Bacterial Loads}

The bacterial load of surface-sterilized individuals was quantified by plating serial dilutions of lysates obtained from each individual fly (from a group of 15 flies) on nutrient agar plates (MRS for L. plantarum). Homogenization of individual flies was performed using Precellys 24 tissue homogenizer (Bertin Technologies, France) and $0.75 / 1-\mathrm{mm}$ glass beads in $500 \mu \mathrm{L}$ of the appropriate bacterial culture medium.

\section{Fly Survival Experiments}

Adult flies were orally infected every 2 days with a solution of Ecc (OD $=200$ or 400$) 5 \%$ sucrose (50/50) which was deposited on a filter disk that completely covered the agar surface of the fly vial. The infected flies were subsequently maintained at $25^{\circ} \mathrm{C}$. Results are expressed as the percentage of living flies at different time points after the first infection. At least 2 tubes of 15 flies were used for each survival assay and 3 replicates of this experiment were done.

\section{Quantitative Real-Time PCR}

RNA from entire flies or specific tissues $(n=12)$ were extracted using an RNeasy Mini Kit (Qiagen, catalog \#74106) with DNAse treatment step (Qiagen, catalog \#79254). Purified RNA (10-200 ng) was reverse transcribed using SuperScript III reverse transcriptase from Invitrogen (catalog \#1810291). Quantitative real-time PCR reactions were performed in triplicate using FastStart Universal SYBR Green Master from Roche (catalog \#04913850001). The amount of mRNA detected was normalized to control rp 49 mRNA values. Normalized data were used to quantify the relative levels of a given mRNA according to cycling threshold analysis $(\Delta \mathrm{Ct})$. Primer information can be found in the supplementary material (see www.karger.com/doi/10.1159/000475771 for all online suppl. material).

PGN Transport in Drosophila Does Not

Require SLC15 Proteins
Statistical Analysis

Prism software (GraphPad) was used for statistical analyses. Our datasets were tested for normality using the D'Agostino-Pearson omnibus test and some of our data did not pass the normality test. Consequently, we used an unpaired 2-way ANOVA (Tukey test) or an unpaired Kruskal-Wallis test.

\section{Results}

\section{Expression Pattern and Mutant Generation of} Drosophila SLC15 Transporters

Our goal was to test the involvement of the Drosophila orthologs of the mammalian SLC15 oligopeptide transporters in mediation of PGN transport and signaling in Drosophila. Phylogenetic analysis showed that the Drosophila genome contains 3 putative SLC15 orthologs, i.e., Yin, CG2930, and CG9444, which, respectively, share 38, 41 , and $32 \%$ identity with SLC15A1, their closest vertebrate ortholog (Fig. 1a, b). Although we do not present evidence that these genes are the functional homologs of the human SLC15 proteins, we collectively call Yin, CG2930, and CG9444 "Drosophila SLC15 transporters" in the rest of this paper. To first characterize these genes, we measured their relative expression levels in various immune competent tissues, i.e., the FB, the midgut (MG) and the Malpighian tubules (MT). Our results indicate that although Yin is not particularly enriched in any of these tissues its expression appears restricted to ectodermal derivatives of the intestinal tract (i.e., the proventriculus and the hindgut; Fig. 2a, b). CG9444 is almost exclusively expressed in MT. CG2930 is more broadly transcribed and enriched in mesoderm-derived gut structures such as the ventriculus, the copper cells, and the posterior MG (PMG), and it is almost completely absent from ectodermal gut derivatives.

To further analyze the involvement of theses transporters in PGN-dependent signaling, we generated null mutants for each of these genes (online suppl. Fig. S1). Whereas a classical knockout technology was used for Yin and CG2930, we opted for an alternative strategy for CG9444, in which 375 bp of genomic DNA (including the translation start site) were deleted and replaced by an attP landing site inserted via homologous recombination (online suppl. Fig. S1a) [30]. Regardless of the strategy used, we were able to generate a complete loss-of-function allele for each SLC15 Drosophila ortholog, as indicated by the lack of mRNA expression in mutant flies (online suppl. Fig. S1b). These mutant flies develop normally to adulthood and do not show any obvious phenotype, except for Yin mutant males that have reduced fertility (not shown).

J Innate Immun 2017;9:483-492

DOI: $10.1159 / 000475771$ 


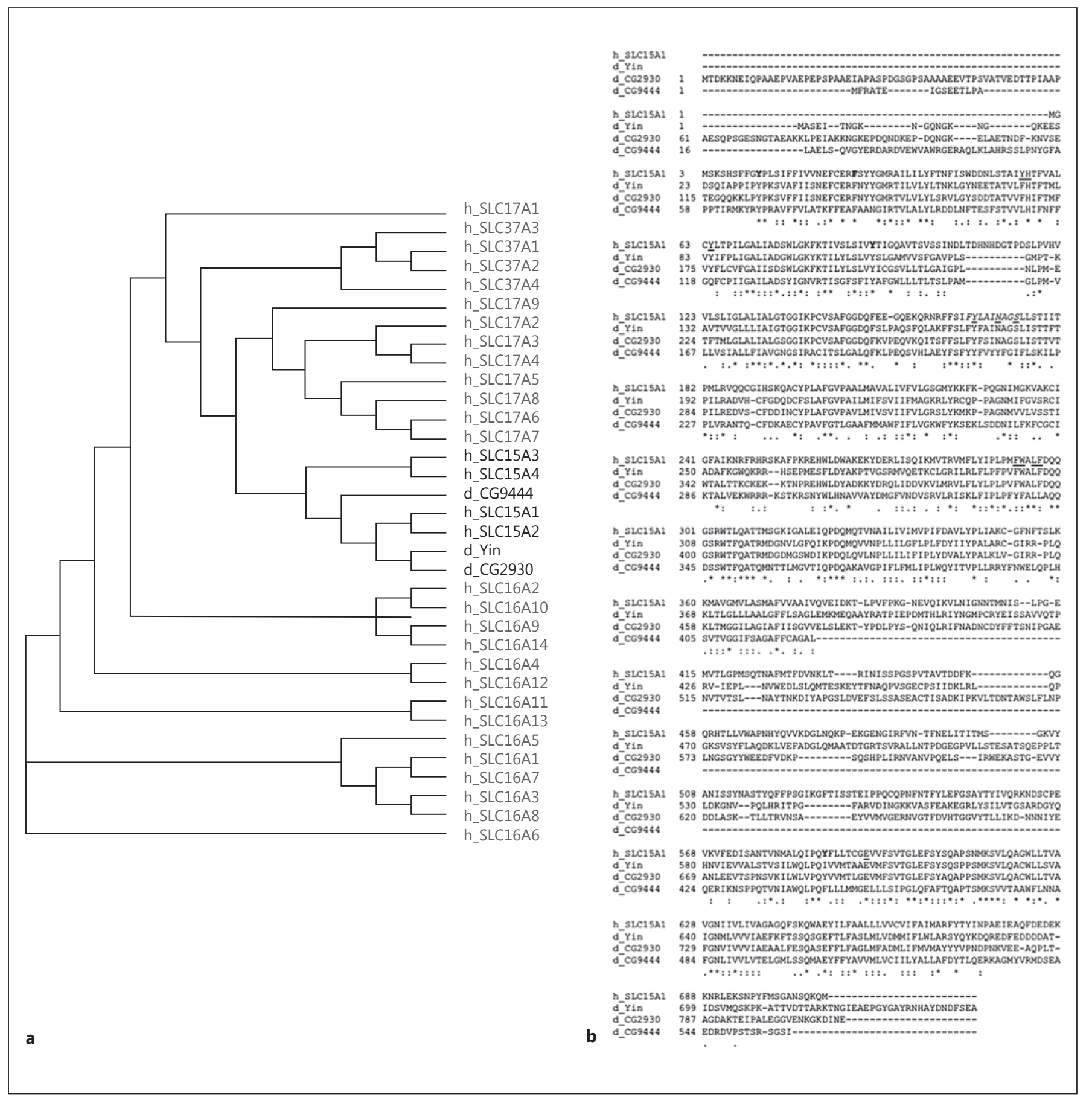

Fig. 1. Drosophila SLC15 vertebrate orthologs. a Phylogenetic tree including the following protein sequences: Drosophila SLC15, human SLC15, human SLC16, human SLC17, and human SLC37. SLC15: oligopeptide transporter family, SLC16: monocarboxylate transporter family, SLC17: organic anion transporter, and SLC37: sugar-phosphate exchanger. This neighbor-joining tree was created using Clustal Omega. b Amino acid sequence alignment of SLC15 transporters. The following 4 sequences were aligned using Clustal Omega: human solute carrier family 15 member 1 (SLC15A1, Uniprot ID: P46059), Drosophila peptide transporter family 1 (Yin, Un- iprot ID: P91679), Drosophila CG2930 (Uniprot ID: Q9W4P6), and Drosophila CG9444 (Uniprot ID: Q9VH93). Asterisks indicate positions that have a single, fully conserved residue. Colons and periods indicate conservation between groups of strongly and weakly similar properties, respectively (see Clustal Omega website for more details). According to Smith et al. [42], underlined amino acids have been identified in human SLC15A1 as essential to the transport function. In bold are the amino acids that play a role in substrate affinity. The amino acid sequence in italics corresponds to a PTR motif involved in proton binding.
Capo/Chaduli/Viallat-Lieutaud/ Charroux/Royet 
Fig. 2. Drosophila SLC15 mRNA levels in immune tissues. a Relative gene expression of Yin, CG2930, and CG9444 mRNAs in axenic adult immune tissues compared to the whole body. $\mathbf{b}$ Relative gene expression of Yin, CG2930, and CG9444 mRNA in different gut domains from axenic adults compared to the midgut. $\mathrm{Pv}$, proventriculus; Vtr, ventriculus; Cc, copper cells; Pmg, posterior midgut; $\mathrm{Hg}$, hindgut. Histograms correspond to the mean \pm SEM of 3 independent experiments. ${ }^{*} p<0.01,{ }^{* * *} p<$ 0.0001 ; ns indicates $p>0.05$ (unpaired 2-way ANOVA vs. a reference value set to 1 for each genotype).

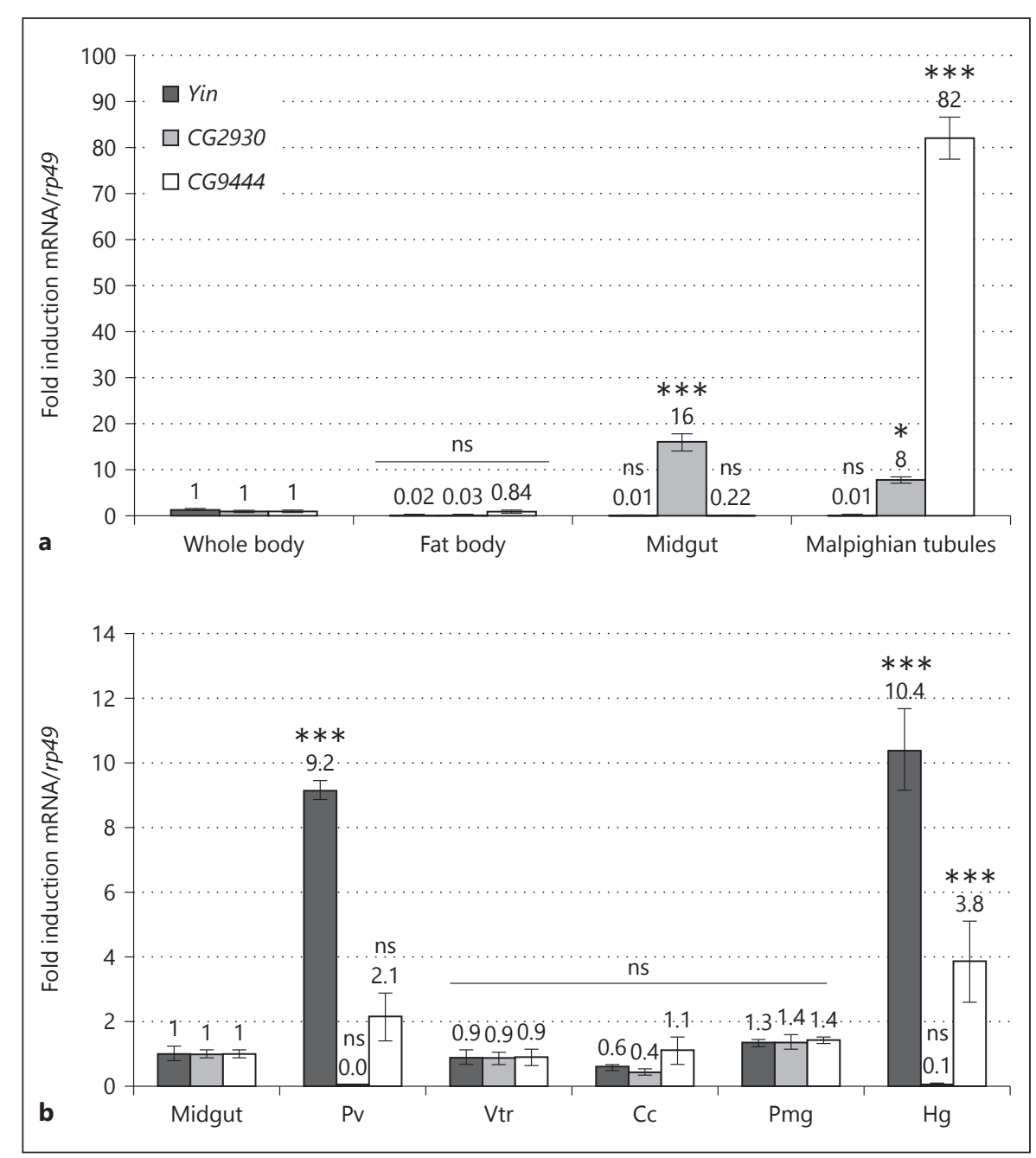

Drosophila SLC15 Mutants Have a Normal

PGRP-LE-Dependent NF- $\kappa B$ Activation

$N F-\kappa B$ activation following bacterial infection in the fly PMG has been shown to be fully dependent on the PGRP-LE cytosolic receptor [24]. Hence, bacterium-dependent transcription of NF- $\mathrm{kB}$ target genes such as Attacin-D, Pirk, PGRP-LB, and PGRP-SC1a/b genes is totally abolished in the PGRP-LE mutant PMG (Fig. 3a; online suppl. Fig. S2). This suggests that, in PMG enterocytes, the PGN has to reach its cytosolic receptor in order to fully activate NF- $\kappa \mathrm{B}$-dependent antimicrobial gene transcription. To test whether any of the 3 SLC15 Drosophila orthologs is involved in PGN delivery to PGRPLE, we monitored NF- $\mathrm{KB}$ target gene expression in the PMG of flies orally infected with the entomopathogenic bacteria Ecc. In contrast to the PGRP-LE mutant in which IMD-dependent PMG activation of Attacin-D,
Pirk, and PGRP-LB transcription was abolished, no obvious modification of $\mathrm{NF}-\kappa \mathrm{B}$ target gene expression was detected when Yin, CG2930, or CG9444 was inactivated (Fig. 3a). The only noticeable phenotype was a constitutive and bacterium-independent upregulation of some NF- $\kappa \mathrm{B}$ targets genes (Attacin-D and Pirk) but not of PGRP-LB in CG2930 mutant PMG (Fig. 3a). Gut bacterial load measurements indicate that the absence of an effect on NF- $\mathrm{kB}$ target gene expression in SCL15 mutants was not a consequence of an increased number of bacteria in the intestinal tract (Fig. 3b). To test whether SLC15 family members display some specificity towards the type of PGN that they might transport, we analyzed the consequences of inactivating Yin, CG2930, and CG9444 on the gut response to the commensal bacterium L.p. As for Ecc, PMG IMD pathway activation (measured by PGRP-SC1 $a / b$ and Pirk mRNA levels) following 


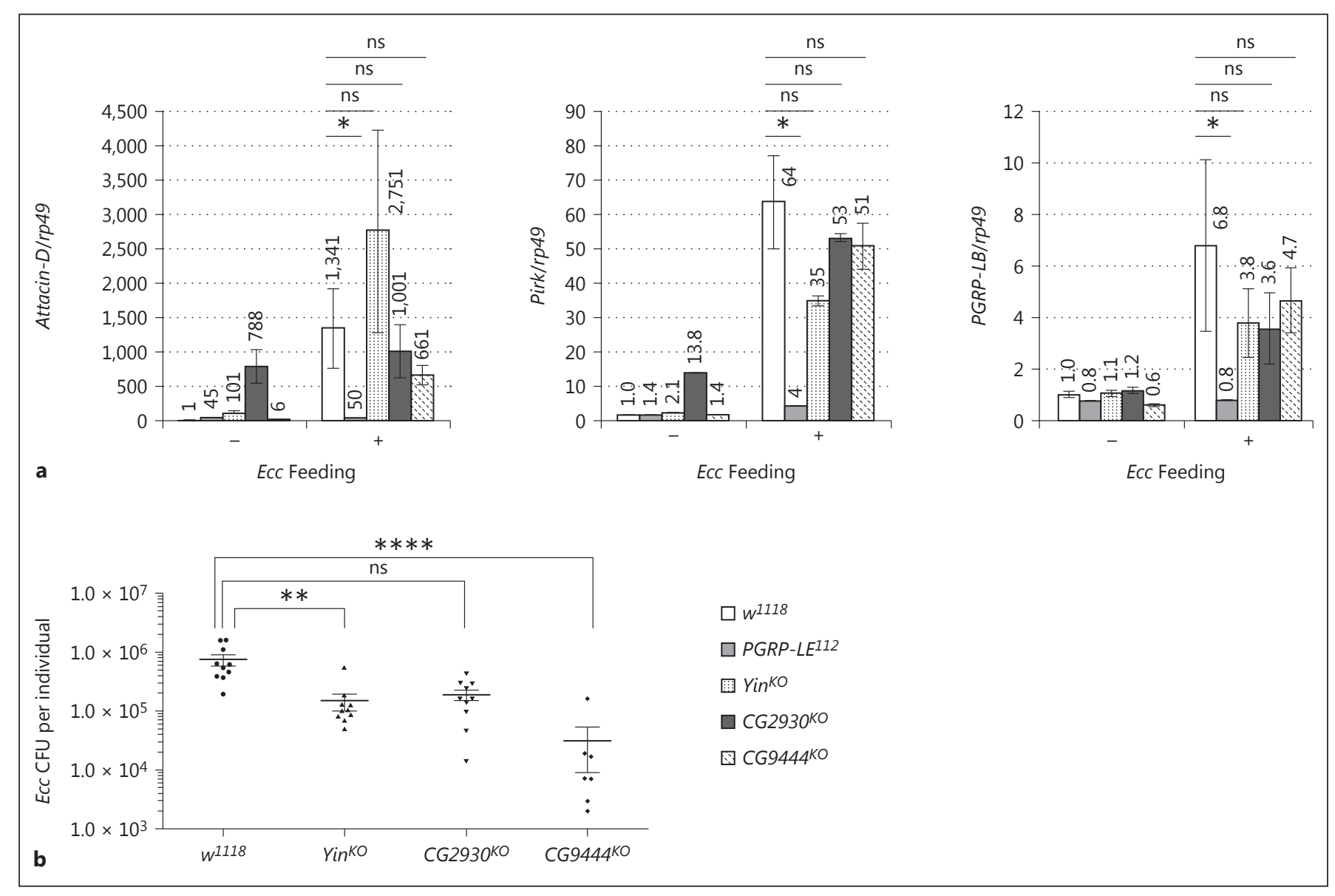

Fig. 3. Drosophila SLC15 transporters are not required for NF- $\mathrm{BB}$ activation in the posterior midgut. a Attacin-D, Pirk, and PGRP-LB mRNA expression in the adult posterior midgut $4 \mathrm{~h}$ after $E c c$ feeding. The mRNA level in axenic control flies was set to 1 , and values obtained with other genotypes are expressed as a fold of this value. Histograms correspond to the mean \pm SEM of 3 independent ex- periments. ${ }^{*} p<0.05$. ns indicates $p>0.05$ (unpaired KruskalWallis test vs. indicated controls). The following genotypes were used: control $\left(w^{1118}\right)$, PGRP-LE mutant (PGRP-LE $\left.{ }^{112}\right)$, and SLC15 transporter mutant flies ( in $^{K O}, C G 2930^{K O}$, and $C G 9444^{K O}$ ). b Bacterial loads of the adult gut $4 \mathrm{~h}$ after Ecc feeding. ${ }^{* *} p<0.01$, **** $p<0.0001$.
L.p. colonization was fully PGRP-LE dependent but it was not affected by elimination of any of the transporters (online suppl. Fig. S2a). However for Yin and CG2930 mutants, we cannot exclude the possibility that the lack of diminution of NF- $\kappa \mathrm{B}$ target transcription is masked by the increased amount of L.p. colonizing the gut (fig S2b). Altogether, these results demonstrate that intracytosolic PGRP-LE does not require the function of either Yin, CG2930, or CG9444 for its activation by bacteriumderived PGN.

Drosophila SLC15 Transporters Are Not Required for PGN Transport from the Gut to the Hemolymph

Previous work in mice has demonstrated that PGN derived from gut microbiota can influence remote tissues and organs [31, 32]. In flies, gut Ecc release PGN that can cross the gut epithelium and its surrounding muscles and reach the hemolymph where it activates the NF- $\kappa B$ pathway in FB cells [33]. Since our previous data demonstrate that PGRP-LE itself is not required for this transport, the mechanisms involved in PGN transport from the gut lumen to the circulating blood remain unknown. To test whether any of the three transporters is involved in such a dialog between tissues, we quantified Diptericin mRNA expression in the FB of flies orally infected with Ecc. In our infection protocol, individual Ecc bacteria are contained within the gut lumen and do not enter the body cavity. Contrary to our expectations, we did not detect a decrease in FB Diptericin mRNA expression in Yin, CG2930, or CG9444 mutants after Ecc oral infection (Fig. 4a). In fact, 


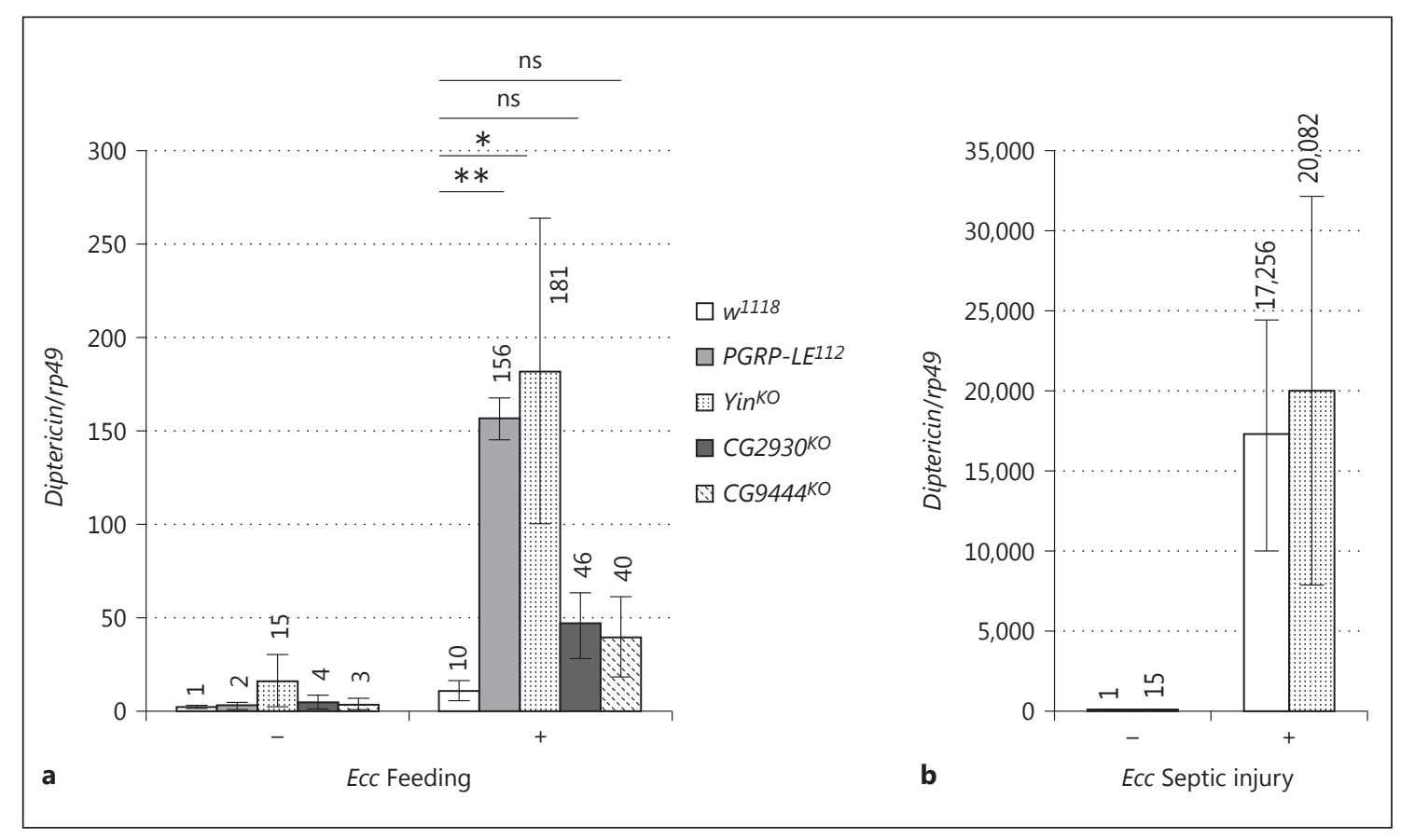

Fig. 4. Increased systemic activation of the immune response in Drosophila SLC15 mutants. Induction level of Diptericin mRNA in the fat body of adult flies $24 \mathrm{~h}$ after Ecc feeding (a) and $6 \mathrm{~h}$ after septic injury with $E c c(\mathbf{b})$. mRNA levels in axenic control flies were set to 1 , and values obtained with other genotypes were expressed as a fold change relative to this value. Histograms correspond to the mean \pm SEM of 3 independent experiments. ${ }^{*} p<0.05,{ }^{* *} p<$ 0.01 . ns indicates $p>0.05$ (unpaired Kruskal-Wallis test vs. indicated controls). The following genotypes were used: control $\left(w^{1118}\right)$, PGRP-LE mutant (PGRP-LE $\left.{ }^{112}\right)$ and SLC15 transporter mutant flies ( Yin $^{K O}, C G 2930^{K O}$, and CG9444 ${ }^{K O}$ ).

in both PGRP-LE and Yin mutants the reasons for this increase are different in the two conditions.

\section{Overactivation of NF- $\kappa B$ Signaling in the FB Reduces} the Life Span of Yin Mutants

Previous work has indicated that overactivation of NF$\kappa \mathrm{B}$ signaling in the FB is detrimental to the fly. The increased expression of Diptericin mRNA in Ecc-fed Yin mutants prompted us to test whether Ecc orally infected flies are short lived [26]. We first showed that inactivation of Yin did not affect the Drosophila lifespan when flies were raised on sucrose medium only (online suppl. Fig. S3a). In contrast, when flies were continuously raised on a medium contaminated with $E c c$, Yin mutant flies (either Yin mutant homozygous or Yin mutant over a deficiency) were the only ones to succumb earlier than the controls (Fig. 5a and online suppl. Fig. S3b). Yin mutant survival curves are very similar to that of PGRP-LE mutants, in which the inactivation of a gut amidase causes PGN accumulation, which in turn leads to detrimental IMD pathway activation in the FB [24]. To test whether the reduced lifespan of Yin mutants in Ecc-contaminated me- 
Fig. 5. Lifespan reduction of Yin mutants after Ecc feeding is due to an overactivation of NF- $\kappa B$ signalling in the fat body. a Survival curves of $E c c$ orally infected flies revealing that YinKO and PGRP-LE112 mutant are more susceptible than CG2930KO, CG9444KO and control flies (w1118). b YinKO susceptibility to Ecc feeding is suppressed when NF- $\kappa \mathrm{B}$ signalling is specifically blocked in the fat body, using CgGal4 driving UAS-dFaddIR. Survival curves are representative of at least three independent trials.

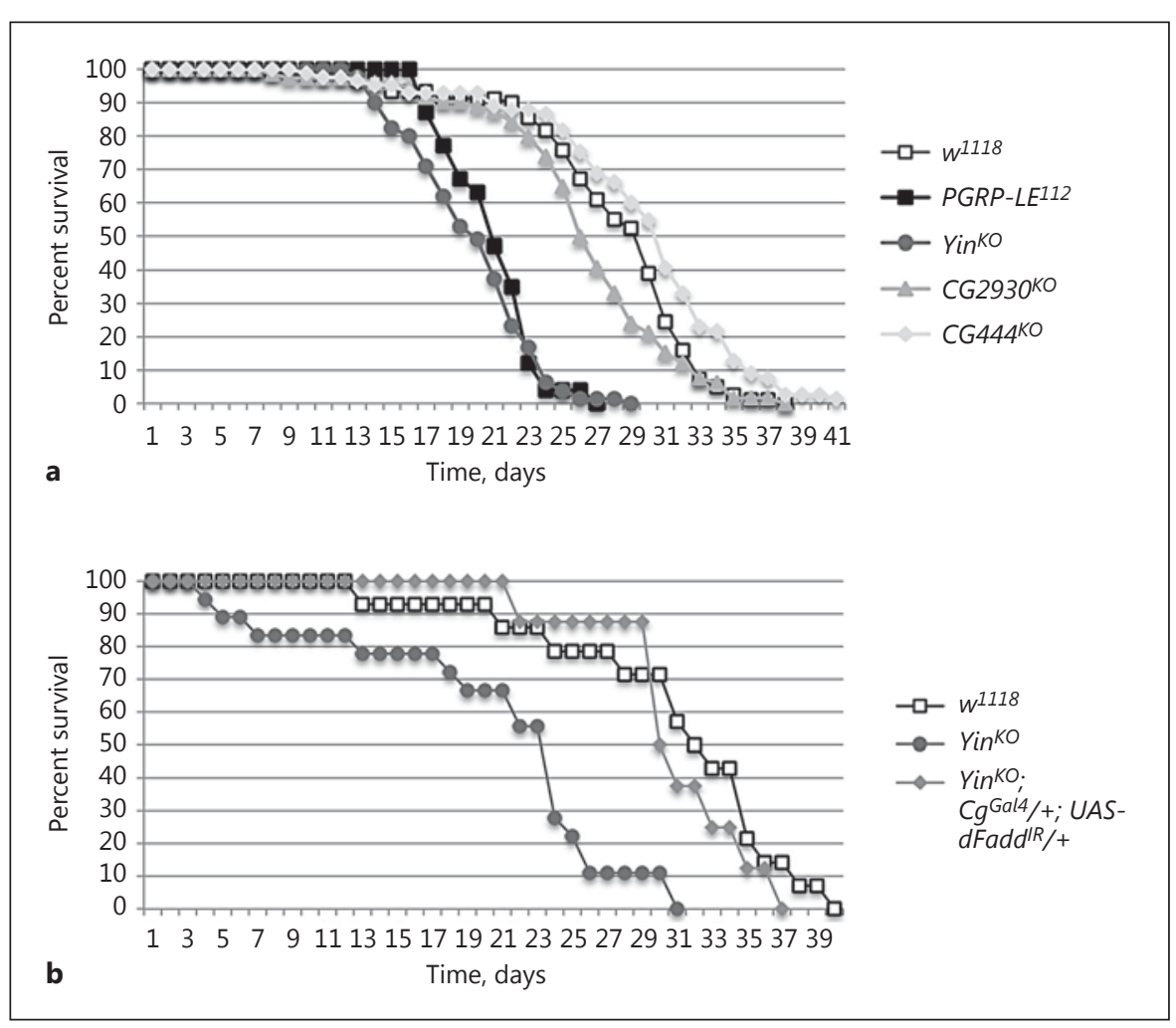

dium was also due to ectopic IMD activation in the FB, we specifically inactivated the IMD pathway (via $d F a d d$ RNAi overexpression) in FB cells. As shown in Figure 5b, inactivation of $d F a d d$ specifically in the FB cells of Yin mutants was sufficient to fully restore their viability. These data demonstrate that Yin function is required to maintain low constitutive IMD pathway levels.

\section{Discussion}

PGRP-LE is an important cytosolic pattern recognition receptor that activates NF- $\mathrm{\kappa B}$ signaling upon bacterial infection. Despite its intracellular localization, PGRP-LE participates in the sensing of extracellular bacteria. The data presented in this report indicate that, in contrast to the situation in mammals, the oligopeptide transporters of the SLC15 family do not seem to be involved in PGN internalization to the cytosol for PGRP-LE sensing in flies. One reason for this discrepancy could be the nature of the PGN-derived ligand, i.e., muropeptides, that might differ between Drosophila PGRP and mammalian Nods [34]. By comparing the activity of natural and synthetic muropeptides, Stenback et al. [35] demonstrated that the minimum active muropeptide for PGRP-LC is GlcNAc-MurNAc-

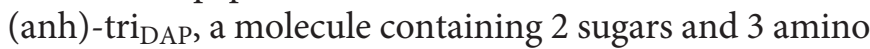
acids. The minimal motif sensed by Nod1 is the tripeptide L-Ala- $\gamma$-D-Glu-meso-DAP, which contains the first 3 residues of a PGN peptide bridge but without the sugar backbone [36]. In contrast, the minimal motif recognized by Nod2 is MurNAc-L-Ala-D-Glu, including the MurNAc residue from the backbone and the first $2 \mathrm{AA}$ of the peptide bridge [36]. This indicates that the minimum active PGN motif required for optimum activation of the PGRP$\mathrm{LC} /$ Imd pathway is larger than those required to activate the Nod PRR. If the different SLC15 proteins have a restricted specificity, they might be able to carry Nod ligands but not PGRP-LE ligands. Consistent with this, previous work has shown that human SLC15A1 transports MDP but no other Nod2-activating molecules, and it does not transport Nod1-stimulating muramyl peptides [37]. Indeed, studies on SLC15A1 and SLC15A2 have demonstrated that these transporters have a much greater affinity for di- or tripeptides than other oligopeptides $[38,39]$. It should also be noted that MDP is not an active ligand for the IMD pathway, and that the exact nature of the ligand able to activate PGRP-LE has not yet been investigated [35]. We have tested here the involvement of the
490

J Innate Immun 2017;9:483-492

DOI: $10.1159 / 000475771$
Capo/Chaduli/Viallat-Lieutaud/ Charroux/Royet 
SLC15 transporters in IMD pathway activation in the PMG, a tissue that contains cells in which IMD pathway activation is mainly dependent on PGRP-LE. It is, however, possible that nonepithelial cells that use PGRP-LE as an intracellular sensor could depend on SLC15 oligopeptide transporters for PGN cytosolic delivery. Finally, it is conceivable that the lack of a phenotype observed in our mutants is due to functional redundancy, a phenomenon that has been observed in mouse macrophages [25].

We observed that the CG2930 mutant presents an increased transcription of some IMD targets in the PMG in axenic conditions. CG2930 transcripts are particularly enriched in the MG, suggesting that it could participate in the active transport of di-/tripeptide is this part of the intestinal tract. The lack of CG2930 might thus result in a less efficient amino acid uptake by the enterocytes and consequently mimics a starvation state which has been described to promote AMP genes transcription via FOXO [40]. We also found that the Yin mutant displays an overactivation of NF- $\kappa \mathrm{B}$ signaling in the FB following Ecc feeding, and that this phenotype is not due to a default of amidases production in the gut. These observations suggest that the amount of PGN circulating in the hemolymph is higher than in control animals. This could result from a more efficient transport of PGN across the gut epithelium or/and from a reduced recycling of PGN once it has reached the hemolymph. Interestingly, studies in mice have indicated that the SLC15A2 transporter is involved in tubular reabsorption of filtered di/tripeptides by the kidneys [41]. We propose that Yin, which is expressed in the MT, could reabsorb the PGN circulating within the hemolymph and will then act as a negative regulator of the immune response.

\section{Acknowledgements}

We thank members of the Royet lab and Jack Green for discussion and comments on this paper. Support for this work was provided by the CNRS, Equipe FRM, to Julien Royet Equipe FRM DEQ20140329541, ANR-11-LABX-0054 (Investissements d'Avenir-Labex INFORM).

\section{References}

1 Motta V, Soares F, Sun T, Philpott DJ: NODlike receptors: versatile cytosolic sentinels. Physiol Rev 2015;95:149-178.

2 Clarke TB, Weiser JN: Intracellular sensors of extracellular bacteria. Immunol Rev 2011; 243:9-25.

3 Viala J, Chaput C, Boneca IG, Cardona A, Girardin SE, Moran AP, et al: Nod1 responds to peptidoglycan delivered by the Helicobacter pylori cag pathogenicity island. Nat Immunol 2004;5:1166-1174.

4 Kaparakis M, Turnbull L, Carneiro L, Firth S, Coleman HA, Parkington HC, et al: Bacterial membrane vesicles deliver peptidoglycan to NOD1 in epithelial cells. Cell Microbiol 2010; 12:372-385.

5 Lee J, Tattoli I, Wojtal KA, Vavricka SR, Philpott DJ, Girardin SE: pH-dependent internalization of muramyl peptides from early endosomes enables Nod1 and Nod2 signaling. J Biol Chem 2009;284:23818-23829.

6 Marina-Garcia N, Franchi L, Kim YG, Hu Y, Smith DE, Boons GJ, et al: Clathrin- and dynamin-dependent endocytic pathway regulates muramyl dipeptide internalization and NOD2 activation. J Immunol 2009; 182:43214327.

7 Vavricka SR, Musch MW, Chang JE, Nakagawa $\mathrm{Y}$, Phanvijhitsiri K, Waypa TS, et al: hPepT1 transports muramyl dipeptide, activating NF-kappaB and stimulating IL- 8 secretion in human colonic Caco2/bbe cells. Gastroenterology 2004;127:1401-1409.
8 Nakamura N, Lill JR, Phung Q, Jiang Z, Bakalarski C, de Maziere A, et al: Endosomes are specialized platforms for bacterial sensing and NOD2 signalling. Nature 2014;509:240244.

9 Royet J, Charroux B: Mechanisms and consequence of bacteria detection by the Drosophila gut epithelium. Gut Microbes 2013;4:259263.

10 Capo F, Charroux B, Royet J: Bacteria sensing mechanisms in Drosophila gut: local and systemic consequences. Dev Comp Immunol 2016;64:11-21.

11 Myllymaki H, Valanne S, Ramet M: The Drosophila imd signaling pathway. J Immunol 2014;192:3455-3462.

12 Buchon N, Silverman N, Cherry S: Immunity in Drosophila melanogaster - from microbial recognition to whole-organism physiology. Nat Rev Immunol 2014;14:796-810.

13 Michel T, Reichhart JM, Hoffmann JA, Royet J: Drosophila Toll is activated by Gram-positive bacteria through a circulating peptidoglycan recognition protein. Nature 2001;414: 756-759.

14 Leulier F, Parquet C, Pili-Floury S, Ryu JH, Caroff M, Lee WJ, et al: The Drosophila immune system detects bacteria through specific peptidoglycan recognition. Nat Immunol 2003;4:478-484.
15 Choe KM, Lee H, Anderson KV: Drosophila peptidoglycan recognition protein LC (PGRP-LC) acts as a signal-transducing innate immune receptor. Proc Natl Acad Sci USA 2005;102:1122-1126.

16 Kaneko T, Yano T, Aggarwal K, Lim JH, Ueda $\mathrm{K}$, Oshima Y, et al: PGRP-LC and PGRP-LE have essential yet distinct functions in the Drosophila immune response to monomeric DAP-type peptidoglycan. Nat Immunol 2006; 7:715-723.

17 Takehana A, Yano T, Mita S, Kotani A, Oshima Y, Kurata S: Peptidoglycan recognition protein (PGRP)-LE and PGRP-LC act synergistically in Drosophila immunity. EMBO J 2004;23:4690-4700.

18 Werner T, Borge-Renberg K, Mellroth P, Steiner H, Hultmark D: Functional diversity of the Drosophila PGRP-LC gene cluster in the response to lipopolysaccharide and peptidoglycan. J Biol Chem 2003;278:2631926322.

19 Ramet M, Manfruelli P, Pearson A, MatheyPrevot B, Ezekowitz RA: Functional genomic analysis of phagocytosis and identification of a Drosophila receptor for E. coli. Nature 2002; 416:644-648.

20 Choe KM, Werner T, Stoven S, Hultmark D, Anderson KV: Requirement for a peptidoglycan recognition protein (PGRP) in Relish activation and antibacterial immune responses in Drosophila. Science 2002;296:359-362.
PGN Transport in Drosophila Does Not

Require SLC15 Proteins
J Innate Immun 2017;9:483-492 DOI: $10.1159 / 000475771$ 
21 Gottar M, Gobert V, Michel T, Belvin M, Duyk G, Hoffmann JA, et al: The Drosophila immune response against Gram-negative bacteria is mediated by a peptidoglycan recognition protein. Nature 2002;416:640-644.

22 Kurata S: Extracellular and intracellular pathogen recognition by Drosophila PGRP-LE and PGRP-LC. Int Immunol 2010;22:143-148.

23 Neyen C, Poidevin M, Roussel A, Lemaitre B: Tissue- and ligand-specific sensing of gramnegative infection in Drosophila by PGRP-LC isoforms and PGRP-LE. J Immunol 2012;189: 1886-1897.

24 Bosco-Drayon V, Poidevin M, Boneca IG, Narbonne-Reveau K, Royet J, Charroux B: Peptidoglycan sensing by the receptor PGRPLE in the Drosophila gut induces immune responses to infectious bacteria and tolerance to microbiota. Cell Host Microbe 2012;12:153165.

25 Charriere GM, Ip WE, Dejardin S, Boyer L, Sokolovska A, Cappillino MP, et al: Identification of Drosophila Yin and PEPT2 as evolutionarily conserved phagosome-associated muramyl dipeptide transporters. J Biol Chem 2010;285:20147-20154.

26 Paredes JC, Welchman DP, Poidevin M, Lemaitre B: Negative regulation by amidase PGRPs shapes the Drosophila antibacterial response and protects the fly from innocuous infection. Immunity 2011;35:770-779.

27 Georgel P, Naitza S, Kappler C, Ferrandon D, Zachary D, Swimmer C, et al: Drosophila immune deficiency (IMD) is a death domain protein that activates antibacterial defense and can promote apoptosis. Dev Cell 2001;1: 503-514.
28 Naitza S, Rosse C, Kappler C, Georgel P, Belvin $\mathrm{M}$, Gubb D, et al: The Drosophila immune defense against gram-negative infection requires the death protein $\mathrm{dFADD}$. Immunity 2002; 17:575-581.

29 Gong WJ, Golic KG: Genomic deletions of the Drosophila melanogaster Hsp70 genes. Genetics 2004;168:1467-1476.

30 Baena-Lopez LA, Alexandre C, Mitchell A, Pasakarnis L, Vincent JP: Accelerated homologous recombination and subsequent genome modification in Drosophila. Development 2013;140:4818-4825.

31 Hergott CB, Roche AM, Tamashiro E, Clarke TB, Bailey AG, Laughlin A, et al: Peptidoglycan from the gut microbiota governs the lifespan of circulating phagocytes at homeostasis. Blood 2016;127:2460-2471.

32 Clarke TB, Davis KM, Lysenko ES, Zhou AY, Yu Y, Weiser JN: Recognition of peptidoglycan from the microbiota by Nod1 enhances systemic innate immunity. Nat Med 2010;16: 228-231.

33 Basset A, Khush RS, Braun A, Gardan L, Boccard F, Hoffmann JA, et al: The phytopathogenic bacteria Erwinia carotovora infects Drosophila and activates an immune response. Proc Natl Acad Sci USA 2000;97: 3376-3381.

34 Chaput C, Boneca IG: Peptidoglycan detection by mammals and flies. Microbes Infect 2007;9:637-647.

35 Stenbak CR, Ryu JH, Leulier F, Pili-Floury S, Parquet C, Herve M, et al: Peptidoglycan molecular requirements allowing detection by the Drosophila immune deficiency pathway. J Immunol 2004;173:7339-7348.
36 Girardin SE, Travassos LH, Herve M, Blanot D, Boneca IG, Philpott DJ, et al: Peptidoglycan molecular requirements allowing detection by Nod1 and Nod2. J Biol Chem 2003; 278:41702-41708.

37 Ismair MG, Vavricka SR, Kullak-Ublick GA, Fried M, Mengin-Lecreulx D, Girardin SE: hPepT1 selectively transports muramyl dipeptide but not Nod1-activating muramyl peptides. Can J Physiol Pharmacol 2006;84: 1313-1319.

38 Fei YJ, Kanai Y, Nussberger S, Ganapathy V, Leibach FH, Romero MF, et al: Expression cloning of a mammalian proton-coupled oligopeptide transporter. Nature 1994;368:563566.

39 Boll M, Herget M, Wagener M, Weber WM, Markovich D, Biber J, et al: Expression cloning and functional characterization of the kidney cortex high-affinity proton-coupled peptide transporter. Proc Natl Acad Sci USA 1996;93:284-289.

40 Becker T, Loch G, Beyer M, Zinke I, Aschenbrenner AC, Carrera P, et al: FOXO-dependent regulation of innate immune homeostasis. Nature 2010;463:369-373.

41 Rubio-Aliaga I, Frey I, Boll M, Groneberg DA, Eichinger HM, Balling R, et al: Targeted disruption of the peptide transporter Pept2 gene in mice defines its physiological role in the kidney. Mol Cell Biol 2003;23:3247-3252.

42 Smith DE, Clemencon B, Hediger MA: Proton-coupled oligopeptide transporter family SLC15: physiological, pharmacological and pathological implications. Mol Aspects Med 2013;34:323-336 